\title{
Comparison Analysis of Donor Liver Volumes Estimated with 3D Magnetic Resonance and 3D Computed Tomography Image Data
}

\author{
Myeong-Seong Kim ${ }^{1,2}$, Kyeong-Seok Park ${ }^{1}$, and Jae-Hwan $\mathrm{Cho}^{3 *}$ \\ ${ }^{1}$ Department of Radiology, National Cancer Center, Ilsan 410-769, Korea \\ ${ }^{2}$ Graduate School of Public Health and Institute of Health and Environment, Seoul National University, Seoul 151-742, Korea \\ ${ }^{3}$ Department of International Radiological Science, Hallym University of Graduate Studies 135-841, Korea
}

(Received 28 July 2014, Received in final form 18 August 2014, Accepted 19 August 2014)

\begin{abstract}
Three-dimensional computed tomography is an effective tool to estimate the liver volume of living donors for the live liver transplantation. When additional operation is required, magnetic resonance imaging is conducted to determine the safety of the donor. This study compared the accuracy of magnetic resonance imaging and computed tomography in estimating 3D liver volume of 23 male and 7 female donors who underwent both magnetic resonance imaging and computed tomography tests before the transplantation. The analysis was conducted to see whether the liver's estimated total volumes and the left lobe volumes obtained from 3Dmagnetic resonance imaging and 3D-computed tomography were identical. Volumes of the right lobe estimated with 3D-magnetic resonance imaging and 3D-computed tomography were compared with the actual volume of the right lobe harvested in the operating room because the volume of the right lobe is an important determinant in the safety of the donor. The total volume of the liver estimated from 3D-magnetic resonance imaging and 3D-computed tomography differed (1238.1904 units and 1402.364 units respectively). The left lobe volume of the liver estimated with 3D-magnetic resonance imaging and 3D-computed tomography also differed (450.530 units and 554.490 units, respectively). The right lobe volume of the liver estimated with 3D-magnetic resonance imaging and 3D-computed tomography were 787.660 units and 847.545 units, respectively, while the actual average right lobe volume of the harvested liver was 678.636 units. 3D-computed tomography has been widely used to estimate the right lobe volume of the donors' liver. However, 3D-magnetic resonance imaging was also very effective in estimating the volume of the liver. Thus, 3D-magnetic resonance imaging is also expected to become an important tool in determining the safety of the donors before transplantation.
\end{abstract}

Keywords : estimation of liver volume, 3D-magnetic resonance imaging for estimation of liver volume, 3D-computed tomography for estimation of liver volume

\section{Introduction}

According to the statistics released by the National Cancer Control Institute of the National Cancer Center, liver cancer was the 5 th most common cancer in Korea in 2011 (incidence rate of 16.463 units). Its 5-year prevalence was 47.698 units, or the 6 th most prevalent cancer in Korea. Among many treatments for liver cancer, liver transplantation is considered the most effective treatment. It is also deemed a good treatment for patients with acute or chronic liver diseases. Gradual increase of liver transplantation has led to increased survival rate. Use of right

CThe Korean Magnetics Society. All rights reserved.

*Corresponding author: Tel: $+82-70-8680-5900$

Fax:+82-2-3453-6618, e-mail: 8452404@hanmail.net lobe graft has become the preferred donor procedure and donors are undergoing diverse imaging examinations. Especially, technologies for estimating liver volume through computed tomography (CT) have been gradually developed. Three-dimensional (3D)-CT images have been widely used to estimate the volume of the liver. Live remnant volume of the donor should be greater than $30-35 \%$ of the total liver volume. In order to avoid small-for-size graft syndrome, the graft-recipient body weight ratio (GRWR) should be more than $0.8-1 \%$ [1]. Also, magnetic resonance imaging (MRI) is used to investigate the donor's blood vessel distribution to determine the safety of the donor. This study investigated liver donors who took received 3D-CT and MRI based on estimation methods used in the National Cancer Center to compare the accuracy of the techniques in estimating 3D liver volume. 


\section{Subjects and Methods}

\subsection{Subjects}

The study involved 30 liver donors ( 23 males, 7 females; 19-50 years of age, average age 29.1 years) who donated their right-lobe graft for liver transplantation between January 2011 and January 2013. Liver volumes were estimated based on CT and MRI examinations conducted before the transplantation and the $3 \mathrm{D}$ volumes were estimated retrospectively.

\subsection{Methods}

MR Systems Achieva Release 3,2,2,0 (Philips Medical Systems, The Netherlands) and CT Brilliance $64 \mathrm{CH}$ (Philips Medical Systems) were used for the examinations. MR Adw 4.2 (GE Medical System, USA) and CT Mx View 3.5 (Philips Medical Systems) were used for the analysis. CT Hepato Biliary Portal Phase and MR eTHRIVE (enhanced T1 high resolution isotropic volume excitation) Portal Phase, which are in use at the hospital, were also used as the protocols. Portal phase parameters are summarized in Table 1. Images obtained during the portal phase of the gadoxetic acid (Primovist)-enhanced MRC (eTHRIVE) were processed in an ADW 4.2 Workstation to estimate the volume of the liver. Reconstructed 5.00 $\mathrm{mm}$ thick images obtained during the hapatobiliary portal phase of the contrast-enhanced CT (Ultravist) were processed in a Mx View Workstation.

Analysis was conducted to see whether the liver's estimated total volumes and the left lobe volumes obtained from 3D-MRI and 3D-CT images were identical. Volumes of the right lobe estimated with 3D-MRI and 3D-CT were compared with the actual volume of the right lobe of the liver harvested in the operating room because the volume of the right lobe is an important determinant in the safety of the donors (Fig. 1). However, the size of the harvested blood-removed liver may shrink because it is cold-stored. Thus, in this study, volume of the big liver vessels was excluded in estimating the size of the liver (Fig. 2). For the analysis of the data, SPSS version 18.0 (SPSS, USA, Chicago) was used for ANOVA to compare the volume of the harvested right lobe and the

Table 1. Scan parameter of portal phase

\begin{tabular}{ccc}
\hline \hline \multirow{2}{*}{ Parameter } & \multicolumn{2}{c}{ Portal Phase } \\
\cline { 2 - 3 } & CT Hepato Biliary & MR eTHRIVE \\
\hline $\mathrm{kVp} / \mathrm{TR}, \mathrm{TE}$ & 120 & $1.6 / 1.3$ \\
Thickness & 3 & 5 \\
FOV & 340 & 350 \\
Injection Volume/Flow Speed & $120 \mathrm{cc} / 4.0 \mathrm{ml}$ & $10 \mathrm{cc} / 1.5 \mathrm{ml}$ \\
\hline
\end{tabular}

estimated volume of the same area obtained from the 3DMRI and 3D-CT images $(p<0.05)$. Paired-samples T test was performed to identify differences between the estimated volumes and the actual volume of the total liver
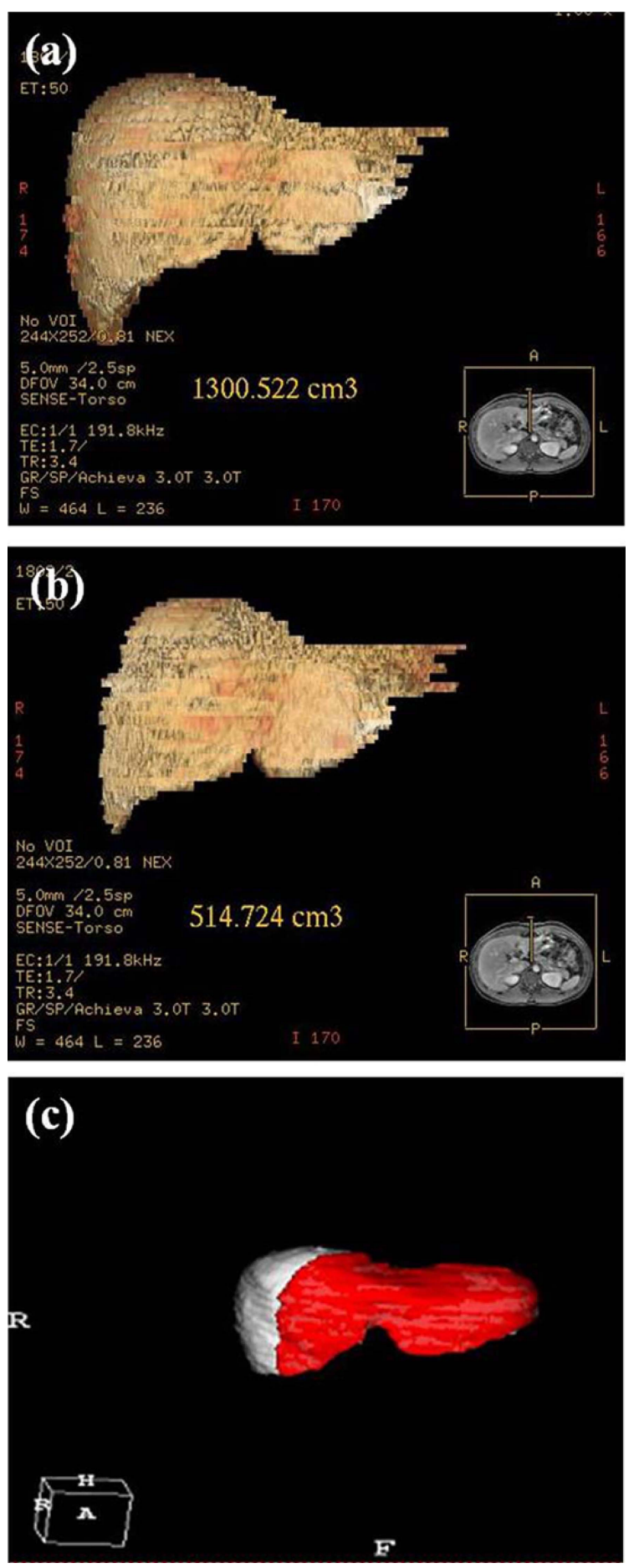

Fig. 1. (Color online) 3D-magnetic resonance imaging total liver volumetry (a) and 3D-MR left lobe volumetry (b) and 3D-computed tomography total liver and left lobe volumetry (c). 

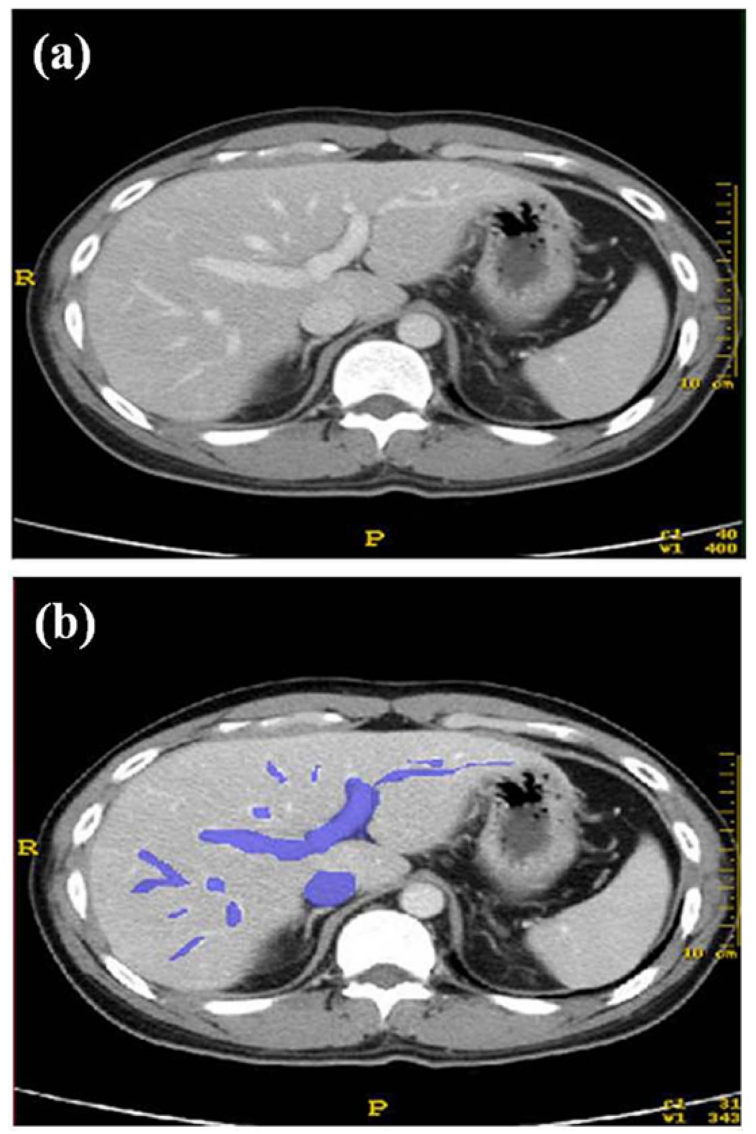

Fig. 2. (Color online) Normal liver image (a) and liver image of excluding blood vessel (b).

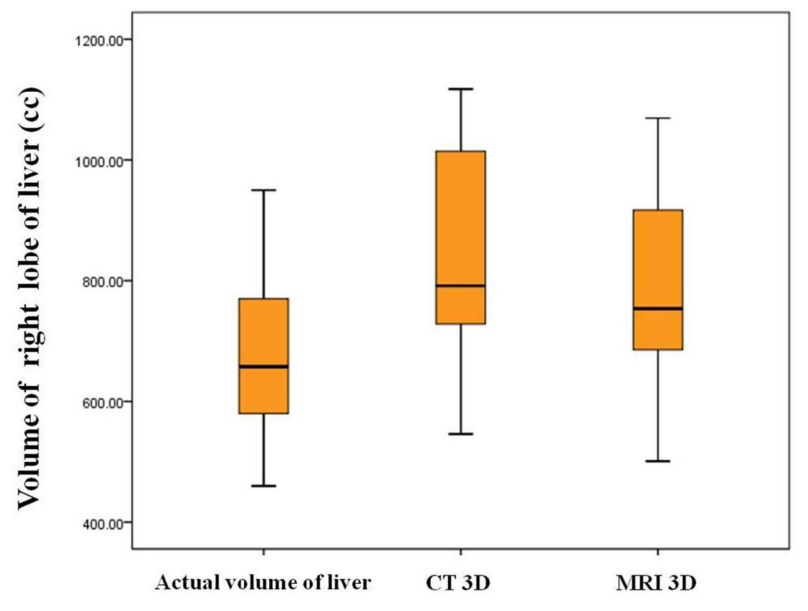

Fig. 3. (Color online) Comparison between the actual volume of the right lobe and the volume of the right lobe estimated with 3D-computed tomography and 3D-magnetic resonance imaging.

and the left lobe $(p<0.05)$.

\section{Results}

Comparison between the actual volume of the harvested liver and the estimated volumes obtained from 3D-CT and 3D-MRI are summarized in Table 2. Actual volume of the right lobe was $678.63 \pm 155.46 \mathrm{cc}$. The volume estimated from the 3D-CT images was the largest (847.54

Table 2. Comparison between the actual volume of the right lobe and the volume of the right lobe estimated with 3D-computed tomography and 3D-magnetic resonance imaging

\begin{tabular}{lcc}
\hline \hline \multicolumn{1}{c}{ Division } & Volume (cc) & P \\
\hline Actual volume of the right lobe & $678.63 \pm 155.46$ \\
Volumes obtained from 3D-computed tomography & $847.54 \pm 177.41$ & 0.00 \\
Volumes obtained from 3D-magnetic resonance imaging & $787.66 \pm 156.35$ & 168.90 \\
\hline Volumes obtained from 3D-computed tomography - Actual volume of the right lobe & 109.02 & 0.02 \\
Volumes obtained from 3D-magnetic resonance imaging Actual volume of the right lobe & 0.04 \\
\hline
\end{tabular}

Table 3. Comparison between the volumes of the whole liver and left lobe estimated with 3D-computed tomography and 3D-magnetic resonance imaging

\begin{tabular}{lc}
\hline \hline \multicolumn{1}{c}{ Division } & Volume (cc) \\
\hline Total volumes obtained from 3D-computed tomography & $1402.03 \pm 268.09$ \\
Total volumes obtained from 3D-magnetic resonance imaging & $1238.19 \pm 231.83$ \\
Total volumes obtained from 3D-computed tomography - Total volumes obtained from 3D-magnetic resonance & $163.84 \pm 87.37$ \\
imaging & $847.54 \pm 177.40$ \\
\hline Left lobe volumes obtained from 3D-computed tomography & $787.66 \pm 156.34$ \\
Left lobe volumes obtained from 3D-magnetic resonance imaging & 0.00 \\
$\begin{array}{l}\text { Left lobe volumes obtained from 3D-computed tomography - Left lobe volumes obtained from 3D-magnetic reso- } \\
\text { nance imaging }\end{array}$ & $59.88 \pm 58.80$
\end{tabular}


$\pm 177.41 \mathrm{cc})$. The differences between the actual volume of the liver and the volumes estimated with 3D-CT and 3D-MR images were $168.90 \mathrm{cc}$ and $109.02 \mathrm{cc}$, respectively. MR volumetry using $3 \mathrm{D}$ image data was closer to the actual volume of the liver. Also, volume of the whole liver estimated with 3D-MR and 3D-CT image data were not identical $(p<0.05)$ (Fig. 3).

Comparison between volumes of the whole liver estimated with 3D-MR and 3D-CT image data are summarized in Table 3. Volume of the whole liver estimated with 3D-CT image was bigger $(1402.03 \pm 268.09 \mathrm{cc})$ than the volume estimated with 3D-MR image data (1238.19 \pm $231.83 \mathrm{cc}$ ). The discrepancy between the two was 163.84 $\pm 87.37 \mathrm{cc}$, showing that the volumes of the whole liver estimated with 3D-MR and 3D-CT image data were not identical $(p<0.05)$.

Comparison between volumes of the left lobe estimated with 3D-MR and 3D-CT image data are summarized in Table 3. Volume of the left lobe estimated with 3D-CT image was bigger $(847.54 \pm 177.40 \mathrm{cc})$ than the volume estimated with 3D-MRI data $(787.66 \pm 156.34 \mathrm{cc})$. The discrepancy between the two was $59.88 \pm 59.80 \mathrm{cc}$, showing that the volumes of the left lobe estimated with 3DMRI and 3D-CT image data were not identical $(p<0.05)$.

\section{Discussion}

In living donor liver transplantation, the safety of the donor operation is the highest priority. The medical team must know for sure that the donor will be safe with the remnant liver volume even after providing enough graft to the recipient. In most adults, volume of the right hepatic lobe accounts for about $60 \%$ of their total liver volume which is enough for the recipients. Thus, identifying the accurate anatomical structure and the volume of the donor's liver became a prerequisite for living donor liver transplantation [2].

Also, medical imaging tests before the transplantation are very important for recipients. Especially, liver volumetry plays an important part $[3,4]$. In this study, 3DMRI and 3D-CT image data were used to estimate volumes of the whole liver, left lobe, and right lobe, which were compared. In the first analysis, volumes of the total liver estimated with 3D-MRI and 3D-CT image were compared: discrepancy of about $170 \mathrm{cc}$ was found between the two. In the second analysis, volumes of the left lobe estimated with 3D-MRI and 3D-CT image were compared: discrepancy of about $100 \mathrm{cc}$ was found between the two. In the third analysis, volumes of the right lobe estimated with 3D-MRI and 3D-CT image were compared with the actual volume of the right hepatic lobe harvested in the operating room: discrepancies of about $100 \mathrm{cc}$ was found between the volume estimated with 3D-MRI and the actual volume; discrepancies of about $170 \mathrm{cc}$ was found between the volume estimated with 3D-CT and the actual volume. Also, the volume estimated with 3D-MRI was about $70 \mathrm{cc}$ smaller than the volume estimated with 3D$\mathrm{CT}$. The reason for the difference between the volumes estimated with the 3D-MRI and 3D-CT images lies in the different technical mechanisms used by each device in obtaining information from the human body. While MRI uses a magnetic field to obtain information of the body from the location of the hydrogen atoms, CT uses radiation to take pictures in a helical fashion. Since CT machines take continuous pictures in a helical fashion, information about tissues may overlap, increasing the volume of the liver more than the volume estimated with the MRI.

Among many medical imaging examinations used to estimate the volume and regeneration of the liver performed before and after the living donor liver transplantation, 3D$\mathrm{CT}$ imaging is one of the most widely used tools, due to the development of multi-detector CT. Especially, 3D volumetry is reported to be most accurate and effective in estimating the volume of the donor's liver [5-9]. 3D volumetry is widely used because it is non-invasive and can delineate the anatomical structure of the blood vessels in the liver. However, in this study the actual volume of the right lobe and the estimated volumes obtained from 3D-MRI and 3D-CT were compared to find if the volume estimated with 3D-MR was closer to the actual volume. Volumes estimated with 3D-MRI were more accurate than the volumes estimated with 3D-CT. The reason for the discrepancies between the volumes estimated with 3D-MR and 3D-CT lies in the fact that $1 \mathrm{~cm}^{3}$ of the liver volume was translated to $1 \mathrm{~g}$ of the liver weight. Other studies have attempted to correct the errors by translating them in 1:1.12 ratio or creating an equation using a correlation coefficient $[1,10]$. Another cause of error is the contraction of the liver after the liver harvesting as the blood is removed from the liver in order to prevent the blood from mixing with the blood of the recipient and is stored at a cold temperature. Removing blood from the liver resulted in much smaller volume [11, 12]. Therefore, examiners of the liver volume should take into consideration the removed blood volume from the liver in estimating the liver volume from the 3D images if they want to enhance the accuracy of the estimation.

\section{Conclusions}

Until now, 3D-CT volumetry of the liver has been 
widely used to estimate the volume of the right lobe of the donor's liver. However, 3D-MR volumetry was also proven in this study to be as accurate and effective as 3DCT. Thus, 3D-MR is expected to become an important examination in determining the safety of the liver donor in the future.

\section{Acknowledgement}

Myeong-Seong Kim and Kyeong-Seok Park equally contributed to this work. They are co-first authors.

\section{References}

[1] P. H. Hayashi, and J. F. Trotter, Aliment Phamacol Ther. 16, 1833 (2002).

[2] M. E. Rinella, E. Alonso, S. Rao, P. Whitington, J. Fryer, M. Abe-cassis, R. Superina, S. L. Flamm, and A. T. Blei, Liver Transpl. 7, 409 (2001).

[3] F. G. Court, S. A. Wemyss-Holden, A. R. Dennison, and G. J. Br, J. Surg. 89, 1089 (2002).

[4] Y. Soejima, M. Harada, T. Suehiro, R. Minagawa, S. Hiroshige, M. Ninomiya, S. Shiotani, and T. Nishizaki,
Surgery. 131, S195 (2002).

[5] P. A. Smith, A. S. Klein, D. G. Heath, K. Chavin, and E. K. Fishman, J. Comput. Assist. Tomogr. 22, 868 (1998).

[6] A. Radtke, G. C. Sotiropoulos, S. Nadalin, E. P. Molmenti, T. Schroeder, F. H. Saner, G. Sgourakis, V. R. Cicinnati, C. Valentin-Gamazo, C. E. Broelsch, M. Malago, and H. Lang, Eur. J. Med. Res. 13, 319 (2008).

[7] N. N. Zein, I. A. Hanouneh, P. D. Bishop, M. Samaan, B. Eghtesad, C. Quintini, C. Miller, L. Yerian, and R. Klatte, Liver Transpl. 19, 1304 (2013).

[8] M. L. Bera, and S. Gupta, Utility of multidetector computed tomography in living donor liver transplantation. Apollo Med. 5, 271 (2008).

[9] T. Yoneyama, K. Asonuma, H. Okajima, K. J. Lee, H. Yamamoto, T. Takeichi, Y. Nakayama, and Y. Inomata, Liver Transpl. 17, 369 (2001).

[10] T. Kiuchi, M. Kasahara, K. Uryuhara, Y. Inomata, S. Vemoto, K. Asonama, H. Egawa, S. Fujta, M. Hayashi, and K. Tanaka, Transplatation. 67, 321 (1999)

[11] H. H. Stone, W. D. Long, R. B. Smith, and C. D. Haynes, Am. Surg. 117, 78 (1969).

[12] S. J. Kim, D. G. Kim, and E. S. Transplant, Proc. 38, 2117 (2006). 